\title{
Electron properties of semiconductor surface studied by the electroreflectance spectroscopy method
}

\author{
P.A. Gentsar, A.I. Vlasenko, A.A. Kudryavtsev \\ V. Lashkaryov Institute of Semiconductor Physics, NAS of Ukraine, \\ 45, prospect Nauky,03028Kyiv,Ukraine,e-mail: gentsar@isp.kiev.ua
}

\begin{abstract}
Relations between the Keldysh-Franz oscillations with electron parameters of semiconductor materials were used to derive qualitative data for homoepitaxial films $n$ GaAs (100) from their electroreflectance spectra. The spectra were measured using the Shottky barrier method at the temperature $300 \mathrm{~K}$ and non-polarized light from the range 1.3-1.65 eV in vicinity of $E_{0}$ transition $\left(\Gamma_{8 \mathrm{v}} \rightarrow \Gamma_{6 \mathrm{c}}\right)$. The spectral data enabled to get values of the following electron parameters: the energy of the electron transition, electrooptical energy, surface electric field, phenomenological parameter of widening, charge carrier relaxation time by energy, relative phase factor, extension of the wave function oscillation and the value of electron mobility. The obtained values are in a good agreement with known data for structurally perfect $n$-GaAs with the electron concentration $n=10^{17}-10^{18} \mathrm{~cm}^{-3}$.
\end{abstract}

Keywords: electroreflectance, Keldysh-Franz effect, homoepitaxial film $n$-GaAs.

Manuscript received 23.09.05; accepted for publication 25.10.05.

\section{Introduction}

In recent years, more and more attention has been paid to surface properties of semiconductors and semiconductor structures [1, 2]. Electroreflectance modulation spectroscopy enables to study electron phenomena inside subsurface layers and to draw conclusions about their structural perfection. The electroreflectance signal is usually observed in the range of direct band-to-band transitions. Therefore, the thickness of the layer providing its formation is determined by the depths of penetration for applied electric field and light. It is related with the fact that geometrical dimensions of operating elements in the last generation of electronic devices have reached the limits when their surface (or surface layers) and interfaces (metal-semiconductor, semiconductor-semiconductor, etc.) begin dominate in their work. Therefore, in this paper analyzed are possibilities of reflectance modulation spectroscopy in studying the electronic properties of semiconductor surface and semiconductor structures using these properties.

\section{Some relations of the electroreflectance theory}

A relative change of a sample reflectivity $\frac{\Delta R}{R}(E, F)$ in the electric field $\mathrm{F}$ can be written as

$$
\begin{aligned}
& \frac{\Delta R}{R}(E, F)=\frac{R(E, F)-R(E, 0)}{R(E, 0)}= \\
& =A\left(n_{0}, n, x\right) \Delta \varepsilon_{1}+\beta\left(n_{0}, n, x\right) \Delta \varepsilon_{2},
\end{aligned}
$$

where $R(E, F)$ is the reflection signal in the case when modulation and constant bias are available; $R(E, 0)$ is the reflection signal when electric field is absent; $E=\hbar \omega$ is the photon energy; $A$ and $B$ are partial coefficients by Seraphin [3]. The presence of the electric field $F$ in semiconductor causes the change of the value of the complex refraction index $N=n+i x$ and, thereof, the value of the complex dielectric function $\varepsilon(E, F)=\varepsilon_{1}(E, F)+$ $+i \varepsilon_{2}(E, F)$ in a semiconductor subsurface area as comparing with its bulk value (Keldysh-Franz effect) [4, 5].

According to $[3,6]$, the change in the imaginary part $\Delta \varepsilon_{2}(E, F)$ of the complex dielectric permittivity for the three-dimensional critical point of 3D $M_{0}$ type in the 
case of direct allowed transitions in the electric field $F$ can be written in the following form:

$$
\begin{aligned}
& \Delta \varepsilon_{2}(E, F)=\Delta \varepsilon_{2}(E, F)-\Delta \varepsilon_{2}(E, 0)= \\
& =\frac{2 e^{2}(2 \mu)^{3 / 2} p^{2}(\hbar \theta)^{1 / 2}}{m^{2} \hbar E^{2}} F\left(\frac{E_{0}-E}{\hbar \theta}\right)= \\
& =\frac{C(\hbar \theta)^{1 / 2}}{E^{2}} F(\eta),
\end{aligned}
$$

where $F(\eta)$ is the electrooptical function of the first kind, the argument of which $\eta$ is equal to

$$
\eta=\frac{E_{0}-E}{\hbar \theta}
$$

where $E_{0}$ is the energy of the electron transition; $\hbar \theta$ is the characteristic parameter inherent to the KeldyshFranz effect (the so-called electrooptical energy) that is equal [3, 6-9]

$\hbar \theta=\left(\frac{e^{2} F^{2} \hbar^{2}}{2 \mu}\right)^{1 / 3}$,

where $e$ is the electron charge; $\mu^{-1}=\left(m_{c}^{*}\right)+\left(m_{v}^{*}\right)^{-1}$ is the reduced effective mass for the optical transition under consideration; $m_{c}^{*}$ and $m_{v}^{*}$ are effective masses of electrons and holes in the conductive and valence bands, respectively; $\hbar$ is the Dirac constant.

The function $F(\eta)$ is determined through the Airy function $A i(\eta)$ and its derivative $A i^{\prime}(\eta)$ in the following form

$$
F(\eta)=\pi\left[A i^{\prime 2}(\eta)-\eta A i^{2}(\eta)\right]-U(-\eta)(-\eta)^{1 / 2},
$$

where $U(\eta)$ is the Heaviside function

$$
U(\eta)= \begin{cases}1, & \eta \geq 0 \\ 0, & \eta<0\end{cases}
$$

The Airy function $\operatorname{Ai}(\eta)$ describes 1D movement of free charge carriers in a uniform electric field of external forces.

The change of the real part of the complex dielectric permittivity in the electric field $F \Delta \varepsilon_{1}(E, F)$ can be found using the Kramers-Kronig relations

$\Delta \varepsilon_{1}(E, F)=\frac{C_{1}(\hbar \theta)^{1 / 2}}{E^{2}} G(\eta)$,

where $G(\eta)$ is the electrooptic function of the second kind, which takes a look:

$$
\begin{aligned}
& G(\eta)=\pi\left[A i^{\prime}(\eta) B i^{\prime}(\eta)-\eta A i(\eta) B i(\eta)\right]+ \\
& +U(\eta) \eta^{1 / 2}
\end{aligned}
$$

where $B i(\eta)$ and $B i^{\prime}(\eta)$ are the modified Airy function and its derivative. It should be noted that $\operatorname{Ai}(\eta)$ and $\operatorname{Bi}(\eta)$ functions are independent solutions of the effective mass equation for an electron-hole pair in the vicinity of the optical transition [7]. As the electroreflectance signal is formed inside the spatial charge region, taking into account the widening $\Gamma$ of the electron transition $E_{0}$ related to the time of energy relaxation for photogenerated charge carriers, which is caused by their interaction with lattice vibrations, impurities, surface defects, the $\eta$ parameter is equal:

$\eta=\frac{E_{0}-\hbar \omega+i \Gamma}{\hbar \theta}=\frac{E_{0}-E+i \Gamma}{\hbar \theta}$.

The expression (9) means that in calculations of electrooptical effects introduced is the complex frequency $\omega^{\prime}=\omega-i \omega_{1}$. Indeed,

$\eta=\frac{E_{0}-\hbar \omega^{\prime}}{\hbar \theta}=\frac{E_{0}-\hbar \omega+i \hbar \omega_{1}}{\hbar \theta}=$

$=\frac{E_{0}-\hbar \omega+i \Gamma}{\hbar \theta}$,

where $\hbar \omega_{1}=\Gamma$.

In the case of small changes of the phase angle $\Delta \varphi$, the real and imaginary components of the dielectric permittivity can be represented in the form

$\Delta \varepsilon_{1}=\frac{1}{2} \gamma \frac{\Delta R}{R}-\delta \Delta \varphi$,

$\Delta \varepsilon_{2}=\frac{1}{2} \delta \frac{\Delta R}{R}+\gamma \Delta \varphi$,

where

$\gamma=\frac{n}{n_{0}}\left(n^{2}-3 x^{2}-n_{0}^{2}\right)$,

$\delta=\frac{x}{n_{0}}\left(3 n^{2}-x^{2}-n_{0}^{2}\right)$.

The phase modulation $\Delta \varphi\left(E_{0}\right)$ is determined by transformation of the electroreflectance spectrum in accord with the Kramers-Kronig relations:

$\Delta \varphi\left(E_{0}\right)=-\frac{E_{0}}{\pi} p \int_{0}^{\infty} \frac{[\Delta R / R(E)] d E}{E^{2}-E_{0}^{2}}$.

Thus, the relative change of reflectivity inherent to the sample studied in electric field $\frac{\Delta R}{R}(E, F)$ can be expressed through the changes of the real and imaginary parts of the dielectric permittivity $\Delta \varepsilon_{1}$ and $\Delta \varepsilon_{2}$, respectively. These changes of imaginary and real parts, in their turn, can be analitically expressed through the electrooptic functions of the first kind $F(\eta)$ (change of the absorption coefficient in electric field) and the 
second one $G(\eta)$ (change of the refraction index in this field) [3], argument of which possesses the form (9). The layer depth that takes part in the electroreflectance signal formation is determined by the depth of electric field penetration (screening depth $L_{\mathrm{D}}$ or $L_{\mathrm{TF}}$ ) as well as light penetration. Therefore, this method is very sensitive to the structure of a thin subsurface layer with the thickness $d$ (depth of light penetration)

$d=\frac{\lambda}{4 \pi N}$.

It is well known that the modulation spectrum $\frac{\Delta R}{R}$ has a sharp peak at the energy of the forbidden gap $E_{0}$ (in the classically forbidden range $\hbar \omega<E_{0}$ ) and some oscillations above $E_{0}$ (classically allowed range of photon energies $\left.\hbar \omega>E_{0}\right)[3,6]$.

The availability of decaying oscillations in the highenergy spectral range, the period of which decreases with the energy growth and strongly depends on the applied electric field, is characteristic for the high-field measurement mode. In this case, when treating the experimental spectra, it is necessary to perform theoretical calculation of electroreflectance curves by using the expanded Airy functions.

In accord with the theory [10], in the high-field measurement mode for the energies $E_{m}$ that correspond to the extrema $\left|\frac{\Delta R}{R}\right|_{m}$, the following equality is valid:

$m \pi=\psi+\frac{4}{3}\left(\frac{E_{m}-E_{0}}{\hbar \theta}\right)^{3 / 2}$

where $m$ is the number of the oslillation; $E_{m}$ is the energy position of the oscillation extremum; $\psi$ is the relative phase factor. The relationship (17) indicates that the slope of the dependency $\frac{4}{3 \pi}\left(E_{m}-E_{0}\right)^{3 / 2}$ on the oscillation number $m$ allows to determine $(\hbar \theta)^{3 / 2}$ and, consequently, the electrooptic energy value $\hbar \theta$. Using this value, one can determine the surface electric field

$F_{s}=\left[\frac{2 \mu(\hbar \theta)^{3}}{e^{2} \hbar^{2}}\right]^{1 / 2}$

From the uncertainty principle for the energy $E$ and time $t(\Delta E \cdot \Delta t \geq \hbar)$, relaxation effects in light absorption by a crystal can be described by the phenomenological parameter of widening $\Gamma$ related with the time $\tau$ of the energy relaxation of photogenerated charge carriers via the following relation $\Gamma=\frac{\hbar}{\tau}$. This relation allows to use the optical method of electroreflectance for estimation of the $\tau$-value for the corresponding electron transitions.
The phenomenological parameter of widening $\Gamma$ describing the experimental spectra is usually derived from fitting the theoretical spectra to the experimental ones. The value of the phase factor $\psi$ is determined using the extrapolation of the straight-line dependence $\frac{4}{3 \pi}\left(E_{m}-E_{0}\right)^{3 / 2}=f(m)$ up to the intersection point with the absciss and the formula (17).

The high-field spectrum enables to ascertain the relation between periods of the Keldysh-Franz oscillations $\Delta E_{m}=E_{m}-E_{m-1}$ and electron parameters of semiconductor materials. It follows from (17) that

$\Delta E_{1}=E_{1}-E_{0}=\left[\left(\frac{3}{4}(\pi-\psi)\right)^{2 / 3}\right] \cdot \hbar \theta$

$\Delta E_{m}=E_{m}-E_{m-1}=$

$=\left[\left(\frac{3}{4}(m \pi-\psi)\right)^{2 / 3}-\left(\frac{3}{4}([m-1] \pi-\psi)\right)^{2 / 3}\right] \cdot \hbar \theta$,

where $m=2,3,4 \ldots$

Besides, it follows from (17) that

$\Delta E=E_{m}-E_{0}=\left(\frac{3}{4}(m \pi-\psi)\right)^{2 / 3} \cdot \hbar \theta$

where $\Delta E$ is the distance from the transition energy to the extremum of the last oscillation.

It should be a relation between these experimental parameters

$\Delta E=E_{m}-E_{0}=2 \frac{(\hbar \theta)^{3}}{\Gamma^{2}}=2\left(\frac{e^{2} F^{2} \tau^{2}}{2 \mu}\right)$.

In the framework of the Keldysh-Franz effect, the characteristic parameter $\lambda_{\mathrm{KF}}$ associated with the extension of the wave function oscillation inherent to a quantum-mechanical particle with the reduced mass $\mu$ being in the homogeneous electric field $F_{s}$ (i.e., the electron wavelength from the energy $\hbar \theta$ ) can be found using the formula

$\lambda_{K F}=\frac{\hbar \theta}{e F_{S}}$.

If experimental electroreflectance spectra do not contain these Keldysh-Franz oscillations, and the electrooptical energy $\hbar \theta$ is comparable with the phenomenological parameter of widening $\Gamma$, then realized is the so-called intermediate case.

It should be noted that in the range of intermediate fields the change of dielectric permittivity $\Delta \varepsilon=\varepsilon(E, F)$ $-\varepsilon(E, 0)$ is approximately linear versus the electric field $F[11]$. We have no possibility for detailed description of the features inherent to experimental spectra obtained in the intermediate fields as well as their informativity, which will be done in future publications. 
In the case $\hbar \theta \leq \Gamma / 3$, one can observe vanishing the Keldysh-Franz oscillations and transformation of the experimental spectrum into the resonance structure consisting of one positive and one negative extrema (the so-called low-field measurement mode). In so doing, informativity of the spectrum is sharply decreased, and the energy of the critical point $E_{0}$ as well as the phenomenological parameter of widening $\Gamma$ are determined using the three-point method [12].

When treating the experimental electroreflectance spectra, one should also take into account the following factors: inhomogeneity of space charge field, dependency of the semiconductor electrostatic potential $V$ on the coordinate $z$ (directed from the crystal surface to its bulk), screening of the applied field by surface states, influence of supplementary mechanisms of charge carrier scattering in the subsurface layer, changes of the band structure as a consequence of changes in physicochemical composition of this layer after various surface treatments (chemical, mechanical, radiation, etc.).

\section{Experimental}

Our measurements of the homoepitaxial $n$-GaAs (100) films with the electron concentration $n=10^{17} \ldots 10^{18} \mathrm{~cm}^{-3}$ were carried out in non-polarized light by using the metal-semiconductor Shottky barrier $[3,13]$ created by deposition of a semi-transparent chromium layer on the surface of studied material.

All the measurements were performed in the vicinity of $E_{0}\left(\Gamma_{8 \mathrm{v}}-\Gamma_{6 \mathrm{c}}\right.$ transition) within the spectral range 1.3 up to $1.65 \mathrm{eV}$. Experimental results were obtained at the room temperature $T=300^{\circ} \mathrm{K}$ and the first modulation harmonic $(f=2.2 \mathrm{kHz})$. The limit relative sensitivity of our measurements was about $5 \cdot 10^{-6}$, and the spectral energy resolution reached $3 \cdot 10^{-3} \mathrm{eV}$.

\section{Results and discussion}

Shown in Fig. 1 is the electroreflectance spectrum for the epitaxial $n$-GaAs (100) film prepared using the method of the Shottky barrier with the electron concentration $5 \cdot 10^{17} \mathrm{~cm}^{-3}$. Polarity of the electroreflectasnce extrema indicates that a depletion layer is created at the surface. To quantitatively interpret the obtained data, one should take into account the availability of decaying oscillations in the high-energy spectral range, the period of which decreases with the energy growth and strongly depends on the applied electric field. These features are characteristic for the high-field measurement mode, therefore, in the figure the experimental results are accompanied with the results of calculations performed using the expanded Airy functions [3, 6] (dotted line). A satisfactorily coincidence with the experimental data has been reached

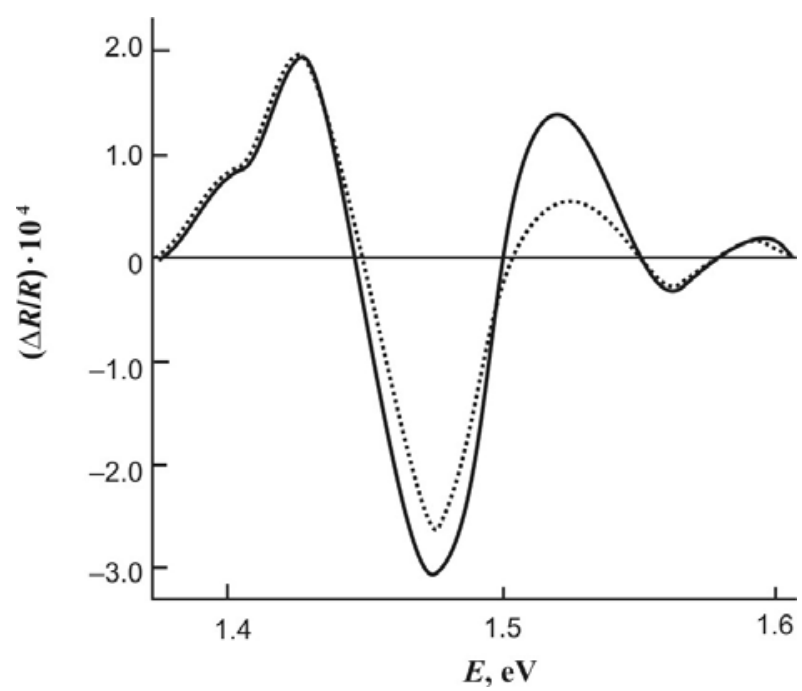

Fig. 1. Electroreflectance spectrum of the epitaxial film nGaAs (100) with the electron concentration $n=5 \cdot 10^{17} \mathrm{~cm}^{-3}$ : solid line - experimental data, dotted line - theoretical fitting for the following parameter values: $E_{0}=1.427 \mathrm{eV} ; \hbar \theta=$ $=0.040 \mathrm{eV} ; \Gamma=0.028 \mathrm{eV}$.

for the following values of parameters: $E_{0}=1.427 \mathrm{eV}$ (energy of the first extremum in the electroreflectance spectrum); $\hbar \theta=0.04 \mathrm{eV} ; \Gamma=0.028 \mathrm{eV}$. In the highenergy spectral range, the observed difference between the fitting electroreflectance curve and the experimental one can be explained by the dependency of the $\Gamma$ parameter on the photon energy $E$.

The parameter $\hbar \theta$ as well as the surface electric field $F_{\mathrm{S}}$ was determined in the following manner. The slope of the dependency $\left(\frac{4}{3 \pi}\right)\left(E_{m}-E_{0}\right)^{3 / 2}$ on the oscillation number $m$ (Fig. 2) yields in the value $(\hbar \theta)^{3 / 2}=0.8 \cdot 10^{-2} \mathrm{eV}^{3 / 2}$. Thereof, $\hbar \theta=0.04 \mathrm{eV}$. The surface electric field $F_{s}$ is equal to $9.8 \cdot 10^{-6} \mathrm{~V} / \mathrm{m}$. When calculating $F_{s}$ by using the formula (18), we took into account the following values of the effective masses for electrons and holes in GaAs: $m_{e}^{*}=0.065 m_{0} ; m_{p}^{*}=$ $=0.475 m_{0}$ [14]. The phase factor value $\psi$ derived from extrapolation the straight line in Fig. 2 and the formula (17) is equal to $\pi / 2$.

In the case of $\psi=\pi / 2$, the formulae (19) to (21) take a look

$\Delta E_{1}=E_{1}-E_{0}=\left[\left(\frac{3 \pi}{8}\right)^{2 / 3}\right] \cdot \hbar \theta$. 
$\Delta E_{m}=E_{m}-E_{m-1}=$

$=\left[\left(\frac{3(2 m-1) \pi}{8}\right)^{2 / 3}-\left(\frac{3(2 m-3) \pi}{8}\right)^{2 / 3}\right] \cdot \hbar \theta$,

where $m=2,3,4 \ldots$

$\Delta E=E_{m}-E_{0}=\left(\frac{3(2 m-1) \pi}{8}\right)^{2 / 3} \cdot \hbar \theta$.

Being based on the formulae (24) and (25), one can obtain the following values $\Delta E_{1}=1.115 \hbar \theta ; \Delta E_{2}=$ $=1.205 \hbar \theta ; \Delta E_{3}=0.94 \hbar \theta ; \Delta E_{4}=0.82 \hbar \theta ; \Delta E_{5}=$ $=0.745 \hbar \theta \ldots$

Based on the asymptotic shape for the high-field limit of the electrooptic functions, the authors of [15] have also shown that the period of the third oscillation is $\Delta E_{3}=0.94 \hbar \theta$. In our measurements, $\Delta E_{3}=0.38 \mathrm{eV}$ for the epitaxial $n$-GaAs film (Fig. 1), which is in a good accordance with the result obtained in [15]. The relations (19) to (21) give the possibility to directly determine the electrooptic energy from the experimental electroreflectance curve. In Table, summarized are the values of $\Delta E_{m}$ and $\hbar \theta$ calculated using the formulae (24) and (25) for the epitaxial GaAs film. This Table confirms validity of these formulae.

Table. $\Delta E_{m}$ and $\hbar \theta$ values inherent to the epitaxial film $n$-GaAs (100) with the electron concentration $n=5 \cdot 10^{17} \mathrm{~cm}^{-3}$.

\begin{tabular}{|c|c|c|}
\hline$m$ & $\Delta E_{m}, \mathrm{eV}$ & $\hbar \theta, \mathrm{eV}$ \\
\hline 1 & 0.045 & 0.04035 \\
\hline 2 & 0.048 & 0.03983 \\
\hline 3 & 0.038 & 0.04042 \\
\hline 4 & 0.033 & 0.04024 \\
\hline
\end{tabular}

The phenomenological parameter of widening $\Gamma$ is found from the comparison of theoretical and experimental electroreflectance curves (Fig. 1). The time $\tau$ for the energy relaxation of photogenerated charge carriers was determined in the following manner. Taking into account the value of $\Gamma$-parameter $\Gamma=0.028 \mathrm{eV}$ for the epitaxial $n$-GaAs (100) film with the concentration of free electrons $n=5 \cdot 10^{17} \mathrm{~cm}^{-3}$, we obtained $\tau=\hbar / \Gamma=2.35 \cdot 10^{-14} \mathrm{~s}$. The distance $\Delta E$ from the transition energy $E_{0}$ to the extremum of the last oscillation in the $n$-GaAs film is close to $\Delta E=0.164 \mathrm{eV}$. The empirical dependency [16] for the electron mobility $\mu_{e}(\Gamma)$ enables to estimate its value in our case as $\mu_{e}=3200 \frac{\mathrm{cm}^{2}}{\mathrm{~V} \cdot \mathrm{s}}$ in the same film.

The light penetration depth calculated using the formula (16) inside the energy range 1.3 up to $1.65 \mathrm{eV}$ changes from $17.27 \mathrm{~nm}$ (for $E=1.65 \mathrm{eV}$ ) up to 21.92 $\mathrm{nm}$ (for $E=1.3 \mathrm{eV}$ ). Here, we took into account the

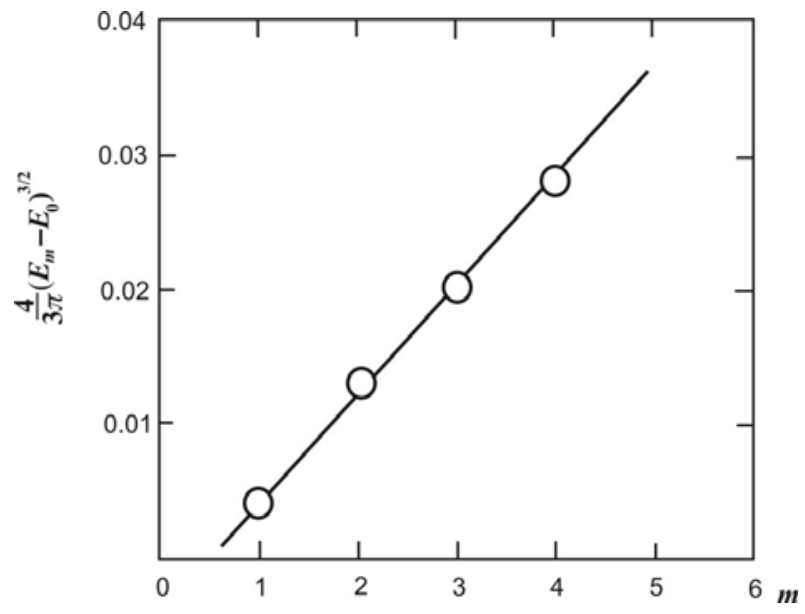

Fig. 2. Dependency of the value $(4 / 3 \pi) \cdot\left(E_{m}-E_{0}\right)^{3 / 2}$ on the number of oscillations $m$ for the epitaxial film $n$-GaAs (100) with the electron concentration $n=5 \cdot 10^{17} \mathrm{~cm}^{-3}$.

value of the dielectric permittivity for GaAs $\varepsilon=12$. The electric field penetration depth can be estimated in accord with [6] using the formula

$L_{\mathrm{D}}=\left(\frac{\varepsilon \varepsilon_{0} k_{\mathrm{B}} T}{e^{2} n}\right)^{1 / 2}$

In our case, $L_{\mathrm{D}}$ equals to $5.758 \mathrm{~nm}(\varepsilon=12$, $\left.\varepsilon_{0}=8.854 \cdot 10^{-12} \mathrm{~F} / \mathrm{m}, k_{\mathrm{B}} T=0.025 \mathrm{eV}\right)$. It is known that the information depth in the studied material is determined by the least value of the penetration depths: by the light penetration one or electric field penetration one. In this case, it should be drawn the conclusion that the information depth is determined by the value of the screening length $L_{\mathrm{D}}$.

Using the above formula (23) and data of Fig. 2, we have also determined the extension $\lambda_{\mathrm{KF}}$ of the wave function oscillation for the mechanical particle with the above reduced mass $\mu$ in the homogeneous electric field $F_{s}$ (Fig. 2). This parameter characteristic for the Keldysh-Franz effect is equal to $4.08 \mathrm{~nm}$.

\section{Conclusions}

The made by authors analysis of relations between the Keldysh-Franz oscillations and electron parameters of semiconductor materials in the high-field measurement mode is used, as an example, to quantitatively treat the electroreflectance spectra of homoepitaxial $n$-GaAs (100) films. The obtained values of their electron parameters, namely: the energy of the electron transition, electrooptical energy, surface electric field, phenomenological parameter of widening, charge carrier relaxation time by energy, relative phase factor, extension of the wave function oscillation and the value of electron mobility, - confirmed structural perfection of these films. Experimental electroreflectance spectra of

\section{(C) 2005, V. Lashkaryov Institute of Semiconductor Physics, National Academy of Sciences of Ukraine}


the films are well described by the single-electron theory if using the obtained values of electron parameters.

\section{References}

1. A.O. Volkov, O.A. Ryabushkin, Microwave-optical modulation spectroscope for studying the semiconductor structures // Pribory $i$ tekhnika eksperimenta No 5, p. 121 - 125 (2001), in Russian.

2. A.A. Gerasimovich, S.V. Zhokhovtsev, G. Gobsh, D.S. Domanevsky, Electroreflectance and reflection of the structure GaAs/AlGaAs with the single quantum well at the room temperature //Fizika $i$ tekhnika poluprovodnikov 39(6), p. 729-734 (2005), in Russian.

3. V.A. Tyagai, O.V. Snitko, Light electroreflectance in semiconductors. Naukova dumka, Kiev, 1980, 302 p.

4. A.A. Evstigneev, O.V. Snitko, L.V. Artamonov, P.A. Gentsar, A.N. Krasiko, Surface contribution to the electroreflectance effect and its separation in the presence of polarization anisotropy // Ukrainskiy fizicheskiy zhurnal 31(5), p. 756-759 (1986), in Russian.

5. A.A. Evstigneev, O.V. Snitko, A.N. Krasiko, P.A. Gentsar, E.V. Mozdor, Studying the mechanically disturbed germanium surface by using the method of anisotropic electroreflectance // Ibid. 32(2), p. 269-272 (1987), in Russian.

6. P. Yu, M. Cardona, Basics of semiconductor physics. Fizmatlit, Moscow, 2002, 560 p. (in Russian).

7. M. Cardona, Modulation spectroscopy. Mir, Moscow, 1972, 416 p. (in Russian).

8. Yu.V. Vorobyov, V.N. Dobrovol'sky, V.I. Strikha, Methods for semiconductor investigations. Vysshaya shkola, Kiev, 1988, 232 p. (in Russian).
9. G.P. Peka, V.I. Strikha, Surface and contact phenomena in semiconductors. Lybid', Kiev, 1992, 238 p. (in Russian).

10. D.E. Aspnes, Band nonparaboliity, broadening and internal field distributions: The spectroscopy of Franz-Keldysh oscillations // Phys. Rev. B 10(10), p. 4228-4238 (1974).

11. O.I. Guzhva, P.A. Gentsar, A.M. Evstigneev, A.N. Krasiko, N.D. Marchuk, T.N. Nikolaeva, O.V. Snitko, V.P. Cherkashin, Optical and electrooptical properties of heavily doped $n-\mathrm{Ge}_{1-\mathrm{x}} \mathrm{Si}_{\mathrm{x}}$ alloys // Fizika i tekhnika poluprovodnikov 21(8), p. 14081412 (1987), in Russian.

12. D.E. Aspnes. Third-derivative modulation spectroscopy with low-field electroreflectance // Surface Science No 37, p. 418-442 (1973).

13. M.B. Guseva, E.M. Dubinina, Physical principles of solid-state electronics. Published by Moscow State University, Moscow, 1986, 312 p. (in Russian).

14. T.S. Moss, G.J. Burrell, B. Ellis, Semiconductor Opto-Electronics. Mir, Moscow, 1976, 431 p. (in Russian).

15. O.Yu. Borkovskaya, S.A. Grusha, N.L. Dmitruk, A.M. Evstigneev, N.A. Klebanova, R.V. Konakova, A.N. Krasiko, K.A. Ismailov, I.K. Sinishchuk, M.E. Lisogorsky, Structural-impurity ordering under action of small doses of penetrating radiation // Zhurnal tekhnicheskoi fiziki 55(10), p. 1977-1982 (1985), in Russian.

16. A.M. Evstigneev, P.A. Gentsar, S.A. Grusha, R.V. Konakova, A.N. Krasiko, O.V. Snitko, Yu.A. Tkhorik, Collision widening of optical spectra and its relation with the mobility // Fizika i tekhnika poluprovodnikov 21(6), p. 1138-1141 (1987), in Russian. 